$\begin{array}{ll}\text { Italique } & \text { Italique } \\ \text { Poésie italienne de la Renaissance }\end{array}$

XIX | 2016

Varia

\title{
Modalità epistolari nei capitoli in terza rima di Lattanzio Benucci (1521-1598)
}

Johnny L. Bertolio

\section{(2) OpenEdition}

\section{Journals}

Edizione digitale

URL: http://journals.openedition.org/italique/433

DOI: 10.4000/italique.433

ISSN: 1663-4438

\section{Editore}

Librairie Droz

\section{Edizione cartacea}

Data di pubblicazione: 1 dicembre 2016

Paginazione: 131-165

ISBN: 978-2-600-04777-7

ISSN: 1423-3983

Notizia bibliografica digitale

Johnny L. Bertolio, « Modalità epistolari nei capitoli in terza rima di Lattanzio Benucci (1521-1598) », Italique [Online], XIX | 2016, online dal 01 décembre 2018, consultato il 07 septembre 2019. URL

http://journals.openedition.org/italique/433; DOI : 10.4000/italique.433 


\author{
J OHNNY L. BERTOLIO \\ M O D A L I T À E P IST O L A R I \\ NEI CAPITOLI IN TERZA RIMA \\ D I L A T T A N Z I O B E N U C C I (1521-1598)
}





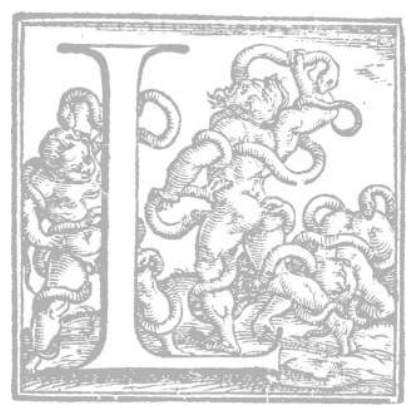

a figura e l'opera di Lattanzio Benucci, letterato e giureconsulto senese di pieno Cinquecento, cominciano a svelarsi in tutte le sue sfaccettature. Citato qua e là nelle raccolte erudite e sempre presente nei saggi su Tullia d'Aragona, che l'ebbe ospite a Firenze e lo rese interlocutore del suo dialogo Della infinità di amore (I547), oltre che di vari scambi in forma di sonetto, Lattanzio ha ricevuto attenzione anche dal punto di vista

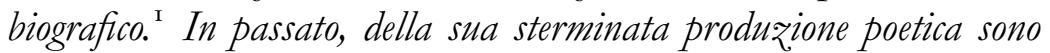
state isolate una serie di sonetti legati a Tullia ${ }^{2}$ e una corona di dieci sonetti caudati raccolti sotto il titolo collettivo della Civetta, una rovente discesa nell'agone letterario a margine della disputa CaroCastelvetro, che vide Benucci schierarsi dalla parte del commendatore marchigiano. ${ }^{3}$

Allineandoci con il tema generale del presente volume ci proponiamo qui di analizzare qualche altro segmento significativo delle poesie di Lattanzio, fermo restando che il Nostro si cimentò, sul versante epistolare puro, in svariate lettere, e non solo in relazione alle sue mansioni di funzionario, tra Roma, Venezia, la Francia e Firenze. Dal punto di vista che maggiormente ci interessa, quello letterario, la penna di Benucci si prodigò in alcune lettere di dedica in apertura delle sue opere in poesia e in prosa: le Elegie, ${ }^{4}$ dedicate in origine a Onorata Tancredi, nobildonna senese e musa petrarchesca di Lattanzio,' quindi alla romana Beatrice Caetani (ca. I546-I600);' una selezione delle sue Rime $^{7}$ a Felice Orsini Colonna (m. Is60); le Osservazioni sopra la Comedia di Dante Aligieri9 e il Dialogo de la lontananza ${ }^{10}$ sempre a Onorata. La presenza femminile, di omaggio devoto e, immaginiamo, sincero (alla Tancredi) o interessato (le romane Caetani e Orsini, possibili patrone in proprio o attraverso $i$ loro congiunti) domina queste testimonianze e asseconda una prassi ben nota agli studiosi e alle studiose di cose rinascimentali.

La vastissima produzione poetica di Benucci impone di lavorare, in questa fase, per saggi e per tal ragione ci si dedicherà ora alla più docile al tema del volume. Per quanto non manchino sonetti di corrispondenza e testi (soprattutto sonettesse e sonetti caudati) polemici e aggressivi, ci soffermeremo sui capitoli in terza rima, indirizzati a un 
destinatario preciso a cui furono probabilmente inviati davvero e che dunque andranno considerati declinazioni in metrica di un'epistola, per quanto spesso giocosa. ${ }^{\text {I }}$

Come diversi letterati del suo tempo, Lattanzio sfruttò in vario modo le potenzialità della terza rima, sia sul versante giocoso sia su quello elegiaco (in linea tematica, e non più solo metrica, con $i$ Triumphi). ${ }^{\mathrm{I} 2}$ I capitoli burleschi di Benucci sono tutti conservati nel codice Magliabechiano Cl. VII 779, autografo e parzialmente danneggiato ${ }^{33}$ (d'ora in avanti F): il titolo onnicomprensivo di Rime, apposto dall'autore, giustifica la presenza di registri molto diversi fra loro, che vanno dal satirico al rusticale, dal petrarchesco allo spirituale. Le Rime sono divise in due libri e tutte le composizioni in questione sono trascritte nel primo, quello decisamente meno allineato con la lezione petrarchesca. Solo tre dei capitoli benucciani sono già stati pubblicati; ${ }^{\mathrm{I}}$ gli altri attendono un'edizione, a cui qui cerchiamo di supplire trascrivendo in appendice $i$ due più interessanti per il nostro discorso. ${ }^{\mathrm{IS}}$

Nel Seicento, sotto il pontificato del senese Alessandro VII Chigi, fu raccolta l'intera produzione letteraria di Lattanzio e F fu trascritto nell'attuale codice Chigi I.VIII.296 della Biblioteca Apostolica Vaticana (da noi siglato $\mathrm{V}_{2}$ ): in esso si ripetono in parte le lacune già presenti in $\mathrm{F}$, ma in qualche caso il manoscritto chigiano permette sia la ricostruqione dei fascicoli danneggiati sia il completamento di alcuni dei capitoli che in $\mathrm{F}$ risultano mutili.

Prima però di qualunque osservazione, sarà utile trascrivere un indice dei componimenti della prima parte di $\mathrm{F}$ che saranno sottoposti ad analisi:

Hora sì, Musa mia, ch'e' ti bisogna (Alla illustrissima Signora la Signora Hersilia Cortese di Monte: cc. Ir-4r);

Fra tante noie che vi stanno appresso (A la nobilissima Madonna Honorata Tancredi: cc. $4 \mathrm{v}-8 \mathrm{r}$ )

S'io potessi ogni volta dar nel segno (All'illustrissima Signora la Signora Hippolita Cibo Sanseverina, Contessa di Gaiazzo: cc. 8v-I Iv)

Non posso più tener la penna a freno (A la nobilissima Madonna Girolama Campani de' Benucci: cc. I 2r-16v)

Poi ch'io son fuor dei libri una mezz'hora (A la medesima [Girolama Campani de' Benucci]: cc. 17 r-25r)

Perch'io v'bo fatta mia procuratrice (A Madonna P. V.: c. $25 \mathrm{v}$, mutilo sia in $F$ sia in $\left.V_{2}\right)$

Forse parravvi cosa strana e nuova (Dell'uova: cc. 30r-33v) 
Signora Tullia, ogn'un che fugge il male (Capitolo de lo spedale. A la signora Tullia d'Aragona: cc. $34 \mathrm{r}-37 \mathrm{v}$ )

Benuccio, a questi di mi fu mandato (Risposta a se stesso in nome de la medesima Signora Tullia: cc. $38 \mathrm{r}-40 \mathrm{v}$, mutilo in $\mathrm{F}$, integro in $\mathrm{V}_{2}$ ) Usanza è stata de gli acuti ingegni (Cavolata contra il cavolo: cc. 56r-62r) Mi sovvien hora haver già un tempo letto (Capitolo del sonno: cc. 62r-65v) Sbracciati a questa volta, o fantasia (Capitolo del sol leone: cc. 66r-69v) Messer Iacomo, poi ch'io son tornato (Capitolo a Messer Iacomo Pecci: cc. $73^{\mathrm{r}-77 \mathrm{r})}$

Essendo voi lontan cotante miglia (Capitolo al medesimo [Iacomo Pecci]: cc. $77 \mathrm{v}-8$ or)

Quel grand'amor che mi poneste in seno (Al Capitano Alessandro Tommasi: cc. $80 \mathrm{v}-85 \mathrm{r}$ )

Questa mattina il buon Tommasi nostro (A Messer Alessandro Tancredi: cc. 8 5r-9or)

S'io havessi la Musa amica tanto ([Al Commun di Bibbiano]: ${ }^{16}$ cc. 9or-94v) Io che sono un ritratto al naturale (Al Signor Teofilo Orsino: cc. 94v-98v) Pavolo e Giulio, signor miei graditi (Dell'amicitia. Al Signor Pavolo di Montoro et al Signor Giulio Acquaviva: cc. 99r-103v)

Se mai mi venne voglia alzarmi a volo (All'illustrissima Signora la Signora Laura $^{17}$ Gonzaga: cc. I04r-I07r)

Si mi trovo accorato dal dolore (A Madonna A. D.: cc. Io7r-I I 2r)

Per farvi un bel segreto aperto e piano (A Madonna Atalanta Donati: cc. I I 5 V-I I $8 \mathrm{v}$ )

Poi ch'io son qui da me stesso lontano (A la magnifica Madonna Verginia Martini: cc. I I 8v+ $12 \mathrm{Ir}$, mutilo in $F$, assente in $V_{2}$ )

Poi ch'è vostra la carta in ch'io vi scrivo (A le sorelle: cc. I 2 I V-I 24V, mutilo sia in $F$ sia in $V_{2}$ )

Perch'io, signora, v'amo da dovero (A la illustrissima Signora la Signora Felice Orsina Colonna: ${ }^{18}$ cc. I48r-I 52 r)

Signor, che per grandezza e per bontade (All'eccellentissimo Signor Duca di Fiorenza. Capitolo in nome de la Signora Tullia d'Aragona: cc. I 5 2r-I 5 v)

Come si vede da un semplice sguardo a titoli e dedicatari, $i$ capitoli di Benucci nascono in seno alla produzione più squisitamente bernesca (s'intenda di Francesco Berni come pure dei suoi emuli), in cui i ternari di impostazione epistolare si affiancano agli elogi paradossali (delle uova, dell'ospedale, del cavolo, del sonno, del solleone) e, almeno nell'ultimo caso (a Cosimo de' Medici), a una circostanza ufficiale. 
Gli elementi che sottolineano la commistione tra la forma poetica del capitolo e quella tipicamente in prosa dell'epistola si dispongono su vari livelli e presuppongono il coinvolgimento attivo del destinatario come lettore, interlocutore e spesso, in quanto noto all'autore, parte in causa rispetto agli eventi e ai temi affrontati nel testo. ${ }^{19}$ Le zone più esposte sono ovviamente i versi di apertura, che seguono direttamente il nome del destinatario citato nella dedica (talvolta più d'uno) o lo ribadiscono, e quelli di chiusura, nei quali il mittente tira le somme di quanto detto e si congeda. Per quanto riguarda gli incipit, semplici vocativi ("Pavolo e Giulio, signor miei graditi», Dell'amicitia, c. 99r; «Perch'io, signora, v'amo da dovero», a Felice Orsini Colonna, c. I48r) possono essere accompagnati da formule di saluto più estese e solenni («Signor, che per grandezza e per bontade / trapassate color che posti in cima / dan legge al mondo in questa nostra etade», a Cosimo de' Medici, c. Is2r). Spesso la parte iniziale contiene il resoconto di un qualche avvenimento che ha attivato la scrittura, proprio come avverrebbe in una lettera in prosa: è il caso dei due capitoli indirizzati all'amico Giacomo Pecci, senese: "Messer Iacomo, poi ch'io son tornato / da Saltennano ${ }^{20}$ a la città di Siena, / mi par che 'l mondo sia tutto rinato» (c. 73r) e "Essendo voi lontan cotante miglia, / né vi scrivendo mai, tengo per certo / vi debbia esser cagion di maraviglia» (c. 77v).

Le indicazioni contestuali si intrecciano con l'iniziativa dello scrivente di mettere mano alla stesura del testo; l'autore fa spesso esplicito riferimento al supporto materiale utilizzato, accostando in modo ancora più diretto la forma del capitolo a quella dell'epistola:

Ma cominciando io voglio anco finire, dico finir d'empirvi almeno il foglio e non finir, ch'io mi voglio morire.

(a Ippolita Cibo Sanseverino, c. 8v)

Ho gran fastidio e senza fin mi doglio non haver tempo a mio modo, ché certo non bastarebbe questo e un altro foglio.

(primo capitolo a Girolama Campani, vv. I 3-1 5, c. I 2r)

È ben ver, quando troppo m'affatico, che la penna mi cade tra le mani, m’avviluppo e non so quel ch'io mi dico.

(a Laura Gonzaga, c. I05r) 
Né Colle o Fabriano ha tante carte ${ }^{21}$ che potessen capire in verso e in prosa le grazie ch'a voi sola il ciel comparte;

$$
\text { (ivi, c. Io6r) }
$$

Per farvi un bel segreto aperto e piano (ch'è di lodarvi e riverirvi ogn'hora) a questa penna mia porgo la mano, laqual so ben ch'in vano hoggi lavora, mentre tenta spiegare i merti vostri in un foglio di carta e in poco d'hora;

$$
\text { (a Atalanta Donati, c. I I 5v) }
$$

Poi ch'è vostra la carta in ch'io vi scrivo, poi ch'è pur vostra questa penna ancora e ch'io del vostro qui mi pasco e vivo, parmi ben dritto d'impiegare un'hora o qualche cosa meno in dir di voi e dell'humor che dentro mi lavora. (alle sorelle, c. I $2 \mathrm{IV}$ )

Le terzine iniziali possono financo diventare sede di dichiarazioni di poetica, come nel primo capitolo che Lattanzio dedica alla madre, poetessa anch'essa, Girolama Campani (vv. I-9, c. I2r):

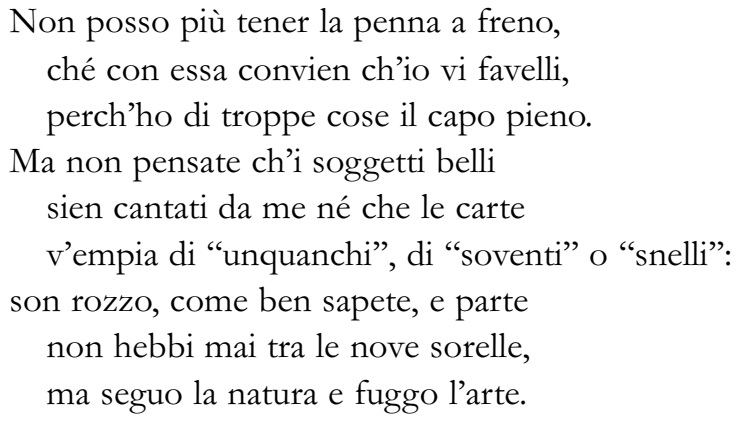

Lattanzio dichiara non solo di voler aderire a uno stile basso ma anche a una lingua fondata sull'uso, e non ricavata da qualche modello imposto a tavolino; appare qui la rivendicazione di uno stile alternativo $e$ insieme una forte autocoscienza linguistica, che emerge anche altrove tra le Rime benucciane. In un componimento contenuto nello stesso codice fiorentino e ispirato in ultima istanza alle teorie linguistiche di 
Benedetto Varchi e Claudio Tolomei, Benucci critica un non identificato «vicino» che, quando deve utilizzare il «toscano» (leggi 'volgare'), attinge alle acque del «Mugnone», mentre al Parnaso preferisce il «Falterona». ${ }^{22}$

Alle terzine finali, se non proprio all'ultimo verso, è invece demandato il compito di sigillare il capitolo con un saluto conclusivo, con un augurio o con indicazioni cronologiche preziose per la datazione: «Nel cinquant'otto, là verso la sera, / da Roma, il dì ch'ogn'uno afferra Agosto» chiude il capitolo Dell'amicitia (un invito ai due destinatari, "Pavolo di Montoro» e "Giulio Acquaviva», a tornare presto in città), che quindi può essere datato al Is agosto I558; tale prassi era particolarmente cara all'Aretino, che alla datazione univa una vera sphraghís apponendo il proprio nome. ${ }^{23}$ La tipica forma di saluto epistolare ('tuo', 'vostro', seguita dalla firma) può essere piegata all'impianto familiare e scherzoso; il capitolo per Felice Orsini si conclude cosi: «sarò sempre vostro e vivo e morto» (c. Is 2 r).

Se non quello dell' autore, il finale può ribadire il nome del destinatario, come nel capitolo in nome de la Signora Tullia d'Aragona (vedi infra) $e$ in quello a Onorata Tancredi (c. $8 r)$ :

Felice me, poi che vivendo in bando del mio nido paterno, io veggo ancora donna tal che, se dritto vo mirando, honorata dal mondo, il mondo honora.

Qui è impiegato un senhal che fa sempre capolino quando c'è di mezzo la donna senese che fu per anni Musa di Lattanzio, il quale amava giocare col suo nome reale come Petrarca con quello di Laura.

Se non il calendario, le terzine finali possono includere l'ora, in genere notturna, entro la quale il capitolo è stato scritto e completato, sottolineando cosi l'esigenza di concludere: "me ne vo in letto a distender le cuoia» (secondo capitolo alla madre, v. 30I, c. 25r); «A Dio, ché suonan le ventiquattr'hore» (secondo capitolo a Giacomo Pecci, c. 8or). Che il capitolo stia terminando è indicato anche da riferimenti metatestuali:

Hor ecco ch'io mi veggo d'esser gionto al fin di questa lunga diceria: però ricido il filo e fo qui ponto (alle sorelle, c. I 24v) 
o da circostanze extratestuali che inducono l'autore a chiudere:

Ma costei lasso e voglio dir hor sopra

i fatti nostri almen trenta parole,

prima che 'l sonno gli occhi mi ricopra.

(primo capitolo alla madre, vv. 85-87, c. I4r)

Ma già mi sento calar dentro a gli occhi

una certa renetta ond'affatica

posso tener che 'l sonno non m'imbrocchi;

(secondo capitolo alla madre, vv. 274-76, c. 24v).

La sollecitazione a 'far punto' può essere dovuta a motivi di tempo; la presenza fisica del servitore dell' amico senese Giacomo Pecci, dal quale l'autore si trova temporaneamente lontano, diventa sprone a chiudere al più presto il capitolo-epistola, cosi da approfittare del suo viaggio di ritorno per la consegna della risposta:

Hor perché il servitor che voi mandate mi sollecita e vuol ch'io lo spedisca, non dirò più, né vi maravigliate;

ch'in vero egli è pur tempo ch'io finisca.

(primo capitolo a Giacomo Pecci, c. $77 \mathrm{r}$ )

Lo stesso accade con un altro amico lontano da Siena: prima il riferimento alla lettera che Lattanzio ha sotto gli occhi ("Hor perch'io son dal tempo molto astretto, / com'ancor voi ne la vostra mi dites, ad Alessandro Tommasi, c. 84r), quindi alla fretta a cui lo ba costretto il messo (ivi, c. 85r):

L'apportator de la vostra m'accuora, ché mi dice ch'io faccia tosto, e certo m'ha dato tempo poco più d'un'hora.

Talvolta, al contrario, l'autore si scusa per essersi dilungato troppo, giustificando la necessità di un resoconto dettagliato con la sapienza dei suoi interlocutori; rivolgendosi con tutta probabilità alla madre, Lattanzio scrive: "Il saper vostro a seguitar m'invita / questa mia cosi lunga diceria, I ma temo non haver la via smarrita〉 (c. 93r), con divertita citazione dantesca. ${ }^{24}$ 
Nel primo capitolo a Girolama, ambientato in un luogo da lei lontano, l'epilogo diventa il surrogato di un saluto filiale, in attesa del ricongiungimento tra $i$ due (vv. I54-57, c. I6v):

Io sanato non so', ma son ben sano, e spero ancor di rivedervi presto e come profumato cortigiano

la man baciarvi: allhor saprete il resto. ${ }^{25}$

Quando Lattanzio rivedrà Girolama potrà non solo riabbracciarla ma anche integrare oralmente quelle informazioni, di natura schiettamente familiare, a cui ha alluso nel corso del capitolo. Nel testo, d'altronde, l'autore aveva già pregato la madre di estendere i propri saluti alle sorelle, che dovevano invece trovarsi vicino a lei (vv. IIs-20, c. Isr):

Intanto voi per dar qualche compenso a quest'assenzia mia, le mie sorelle salutate per me con ogni senso.

Ditele ch'io vorrei saper novelle di loro e che mi scrivin qualche volta, né mi curo che sian parole belle.

Qualcosa di simile avviene con l'amico Pecci, della cui famiglia Benucci vuole avere notizie:

La mia affezzione anco mi muove a dimandar di tutta casa vostra, per saper in che termine si trove. (secondo capitolo a Giacomo Pecci, c. 79r)

Questi riferimenti a persone 'terze' entrano nel testo in quanto rese presenti alla mente dell' autore attraverso l'epistola a cui si sta replicando:

Mentre scrivevo questa, m'è venuta una vostra a le mani e volentieri vi potete pensar ch'io l'ho veduta: se non c'ha intorbidati i miei pensieri l'udir come l'amico infermo sia, che sì gagliardo lasciai pur l'altrhieri;

(primo capitolo a Giacomo Pecci, c. 76r) 
e poco sotto:

Ho letto ne la vostra a questo appresso

che n'andaste là verso Castelnuovo

e per me volentier faceste il messo.

(ivi, c. $76 \mathrm{v}$ )

Dato che Benucci dice di avere già iniziato la stesura del proprio ternario prima di ricevere la missiva di Pecci non si può pensare ad essa come al testo a cui si risponde in versi; tuttavia, la lettera ha indirizzato il mittente su temi non inizialmente previsti, condizionando la replica poetica o, se non altro, la sua parte finale.

Con amici e parenti l'autore sembra quasi avido di fornire notizie di ogni sorta, tanto che $i$ fatti riportati si affastellano in modo caotico mettendo in pericolo il filo logico del discorso; a questo punto, quindi, si rende necessaria una specie di 'proemio al mezzo' per mettere in guardia l'interlocutore e richiamarne di nuovo l'attenzione:

Hor io vo' fare un'altra giravolta e, lassando da parte i casi nostri, tesser una gran tela c'ho ravvolta.

Perciò date voi tregua ai pensier vostri e prestatemi tutta l'attenzione, se volete ch'a pieno io ve la mostri.

(primo capitolo alla madre, vv. I 2 I-26, c. I 5 r)

Anche la rete di conoscenze talvolta si intreccia e coinvolge più persone nella stesura di un capitolo:

Questa mattina il buon Tommasi nostro

$m$ 'ha mostrata una lunga filastrocca

e dicemi ch'ell'è cosa di vostro;

e perché il peso del risponder tocca

a lui et ei non ha la Musa amica,

se non quando l'humor talvolta scocca,

mi stimola ch'io sia quel che vi dica

quattro parole sopra dell'amore

e de la vostra e sua dolce nimica.

(ad Alessandro Tancredi, c. $85 \mathrm{r}-\mathrm{v}$ ) 
Questioni amorose sono al centro di questo capitolo nel quale Lattanzio si fa portavoce di Alessandro Tommasi: questi vorrebbe rispondere alla «filastrocca» (ovvero uno sproloquio di non elevata ispirazione, se non un capitolo vero e proprio) ${ }^{26}$ di Tancredi ma lascia che a farlo sia il meglio attrezzato Benucci.

Chiamate in causa possono essere anche missive già note al destinatario $e$ dedicate al medesimo argomento di cui l'autore si sta occupando nel capitolo:

Io ne saprei contar cose stupende, ma perch'altrove, come voi sapete, n'ho scritto, questo basti a chi l'intende; voi queste insieme con quelle aggiugnete.

(primo capitolo alla madre, vv. 79-82, c. I 4r)

Sulla scrittura che si dipana di terzina in terzina anche in virtù del carattere aperto e potenzialmente infinito della forma capitolo, l'autore riflette legittimando il paragone con un testo a stampa: nella terzina "Già v'hebbi per un gioven molto accorto, / ma hora il testo mio canta altrimenti, / qual è di stampa d'Aldo, e dice "scorto"》 (ad Alessandro Tancredi, c. 87v), l'allusione divertita ai refusi delle coeve edizioni aldine diventa un pretesto per sottolineare la perdita di senno dell'amico, che necessita ora di accompagnamento.

Il carattere volutamente familiare dei capitoli benucciani emerge, soprattutto in quelli indirizzati a parenti e amici, prima di tutto a livello linguistico: se infatti un certo gusto linguaiolo, basato su idiotismi toscani ${ }^{27}$ e potenziato dalle allusioni di natura sessuale, è del tutto prevedibile negli elogi paradossali, ${ }^{28}$ anche nei capitoli epistolari domina un tono medio-basso, verso cui indirizzano vari elementi lessicali e stilistici. Tra questi spicca l'uso di espressioni idiomatiche quando non proverbiali, consapevolmente adoperate («sapete ch'io scherzo volontieri / e m'è questo burlar grato e soave», secondo capitolo alla madre, $v v .245-46$, c. 23v), delle quali già per altri autori è stato notato l'impiego in senso anticlassicistico: ${ }^{29}$ "iio vi rispondo / ch'io sto in cervello e non mi son perduto» (primo capitolo alla madre, $v v$. III-I2, c. Isr); «So ben che voi direte che venuto / mi sia nel capo il grillo» (secondo capitolo alla madre, vv. 7-8, c. I7r); «ch’oltra la mala Pasqua, harò il mal anno» (ivi, v. 72, c. Ior); "Non vo' contra il dover mai fare il callo» (ivi, v. I42, c. 2Ir); "Ma poi ch'ogni piacer mi si disdice, / vi 
ricordo impariate a le mie spese, / come 'l volgar proverbio ogn'bor vi dice» (ivi, vv. 202-4, c. 22v); "Vo' dir che tal hor vengan certi fumi / al cervello e bisogna in ogni modo / ch'ei dia la volta o veramente sfumi» (ad Alessandro Tancredi, c. 86v); «perch'ordinariamente il ciel sereno / non piove mai» (ibid.); «Bisogna caminar col piombo ai piei» (ibid.); «se non fusse che gatta ci cova» (a Laura Gonzaga, c. Iosr); «crepi d'invidia pur chi vuol crepare» (ivi, c. Io6v); «io non posso / starmi più queto, che mi crepa il cuore» (a A. D., c. Io7r); «bisogna star saldo più d'un muro, / far di mercante orecchie a tutto pasto» (ivi, c. IOTv); «se dolore havessi al danno eguale / ci lassarei la pelle non che il pelo» (ivi, c. IIov); "tal vede la brusca in l'occhio altrui / mentre la trave il suo vedere appannas (ivi, c. IIIv: qui massima evangelica); «ben conosco l'uva dall'agresto», ovvero dall'uva acerba (a Felice Orsini Colonna, c. Isov).

Le similitudini, in particolare, sono il terreno privilegiato per assecondare il tono quotidiano dei testi, anche quando questi sono pensati come encomiastici: «preso al boccon, come la lasca all'esca» (a Onorata Tancredi, c. 5v); «come medicina / cacciarla giù» (primo capitolo alla madre, vv. 59-60, c. I3v); " hhor mi vo' star queto come l'oglio» [sic] (secondo capitolo alla medesima, v. 60, c. 19v, con uso di un'espressione che è anche idiomatica); "gli amici, che son senza / voi come la minestra senza il sale» (Dell'amicitia, c. I03r); «come se mi fusse un lupo appresso, / perdo la voce e mi s'arriccia il pelo» (alle sorelle, c. I23r), immagine, questa seconda, presente anche in «hor qui convien che mi si drizzi [lezione originaria: 's'arricci'] il pelo» per significare uno slancio lirico richiesto dal soggetto (a Atalanta Donati, c. II $6 v$ ).

Anche situazioni tipicamente liriche come l'amore per una donna o l'elogio delle virtù delle destinatarie femminili si piegano al tono familiare e scherzoso: "Io so' spolpato et ogn'bor più mi cuoco» (secondo capitolo alla madre, v. 6I, c. I8v); "dite se com'a me sempre v'accade / di star a pollo pesto» ${ }^{30}$ (primo capitolo a Giacomo Pecci, c. 75r; e cfr. "questo non è mal da pollo pesto», a Felice Orsini Colonna, c. ISOv); «So ben ch'io porto fiori a un lieto aprile, / le stelle accresco al ciel quand'è sereno / e cornacchioni aggiungo al campa-

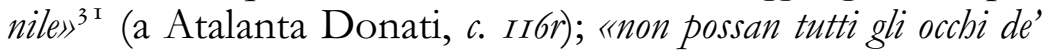
mortali / veder di voi l'alta virtute a pieno, / se ben havesser mille e mille occhiali» (a Felice Orsini Colonna, c. I48r); «pur di nuovo il becco immollo» (ivi, c. ISIV). ${ }^{32}$ 
Gli inserti di formule latine, se in un'epistola familiare e in generale in un'opera in prosa sembrano ben adattarsi al dettato di autori perfettamente bilingui, nei capitoli burleschi non sono affatto comuni ${ }^{33}$ e rientrano in un gioco allusivo tra autore e destinatario: "plusquam perfetto» (a Onorata Tancredi, c. 7 r); «lo stento amore Dei» (secondo capitolo alla madre, v. 44, c. I8r); "quei che si van frustando a tutte l'hore / dietro all'armorum» (a Tullia d'Aragona, c. 34v); «il mio pegno più caro, / idest il cuor» (a Teofilo Orsini, c. 97r); «vorrei veder mantenervi da bene / in ogni tempo et in utroque foro» (ivi, c. 98r); «altro non posso darvi / in scrittis et a bocca che parole» (Dell'amicitia, c. 99r). In quasi tutti questi esempi si tratta di formule da linguaggio cancelleresco e, ben lungi dall'elevare il tono come nel periodare vernacolo di un Machiavelli, creano uno stridente contrasto tra le situazioni ufficiali e formali che evocano e il contesto quotidiano in cui sono inserite.

Un discorso a parte va fatto per il capitolo in nome de la Signora Tullia d'Aragona indirizzato all'illustrissimo et eccellentissimo Signor Duca di Fiorenza (cc. Is2r-IS5v), tra i pochissimi già editi. Con questo testo Lattanzio si espone in prima persona, e a quanto pare in modo ufficiale, in merito a un'ordinanza di Cosimo del I546, in base alla quale tutte le cortigiane dovevano attenersi ad un abbigliamento sobrio, evitare l'uso di gioielli e indossare un velo giallo. ${ }^{34}$ Tullia, che in quel momento si trovava a Firenze da Siena, dove aveva contratto regolare matrimonio, ${ }^{35}$ fu personalmente invitata al rispetto della prescrizione e, desiderosa di imporre la sua fama di letterata, chiese aiuto all'amico Benedetto Varchi per ottenere un'esenzione. Ne scaturi una supplica alla duchessa Eleonora e una raccolta manoscritta di sonetti di vari autori, tra cui Lattanzio, indirizzati a Tullia, identificata da Bausi nell'attuale Magliabechiano VII II $853^{36}$ l'operazione ordita a quattro mani ebbe successo e Tullia ottenne la sperata dispensa nel maggio del I547, nello stesso anno in cui furono pubblicate le sue Rime, con dedica alla medesima Eleonora, e il Dialogo della infinità di amore.

La Tullia del Benucci insiste sulla sua nuova vita, ormai lontana dai piaceri della carne e anzi feconda di incontri intellettuali, secondo quella stessa linea difensiva perseguita d'intesa col Varchi. Rispetto ai capitoli indirizzati ad amici e familiari, questo per Cosimo de' Medici enfatizza l'ineffabilità del soggetto, applicando alle terzine le consuetudini 
retoriche forensi (esordio con lunga captatio benevolentiae, narrazione del fatto in questione, argomentazioni e controargomentazioni, peroratio finale), tanto più in presenza di un destinatario cosi illustre; tuttavia, anche in questa occasione Lattanzio non lesina dettagli quotidiani, come quando la sua Tullia spiega che, a causa dell'ordinanza, non può accogliere adeguatamente in casa i suoi insigni ospiti (c. I54r-v):

[...] s'io non posso arditamente usar le mie bagaglie, son forzata chiuder la porta in fronte a tutta gente

e starmi il più del tempo ritirata, per non esser trovata in farsettino, come s'il pan facessi o la bucata.

Le sue argomentazioni sono sostenute dalla saggezza paremiografica, adibita a rafforzare la forza logica ed esemplificativa del ragionamento (c. Is5r):

Dice un proverbio: chi mattino e sera veste drappo, o gli è ricco o gli sta male; lassa, il mio luogo è in quest'ultima schiera.

Per non irmene dunque a lo spedale, bisogna strologar a tutte l'hore e per fuggire il peggio havere il male.

Il capitolo termina con il nome della donna, che sigilla cosi la promessa di passare il resto della vita cantando le lodi di Cosimo (c. I5sv):

Hor se Dio e natura e virtù tanto vi dier (ché, come il chiaro grido suona, portate solo d'ogni gloria il vanto) esaudite, signor, Tullia Aragona.

Dietro questa immagine pubblica che Tullia e $i$ suoi sodali si sforzarono di coltivare per allontanare il biasimo dei benpensanti si consumava, tuttavia, una prassi letteraria giocosa e burlesca di ben altro tenore. Lo stesso codice fiorentino di Lattanzio, infatti, contiene due capitoli, uno indirizzato a Tullia e l'altro di risposta a se stesso in nome di lei, aventi per oggetto un famigerato «spedale» (cc. 34r-37v e 38r-40v, stessa parola già evocata da Tullia nel capitolo a Cosimo), in cui Bausi ba individuato una serie, tipica di quel genere, di metafore sessuali; ${ }^{37}$ l'elogio 
paradossale si arricchisce cosi di una impostazione epistolare, tanto più che, per quanto nella finzione letteraria, alla "proposta" di Lattanzio segue una pretesa "risposta" della donna, ${ }^{38}$ che inizia con un richiamo al supporto materiale del testo: «Benuccio, a questi di mi fu mandato / un capriccio si bel de lo spedale, / che spesso il leggo e sempre il porto allato» (c. $38 r)$. La risposta si conclude con un invito a far tesoro dei precetti 'ospedalieri' e con un tenero ricordo di Penelope, ufficialmente sorella, e forse figlia, di Tullia, nel cui nome, evocato sempre da Lattanz̧io, finisce anche il Dialogo della infinità di amore :

Hor fate sì ch'io non favelli al vento:

Penelope v'aspetta e vi dimanda;

lo spedal tutto spera esser contento

per le man vostre e vi si raccomanda. ${ }^{39}$

In ultima analisi, $i$ capitoli benucciani, con la loro esuberante vocazione narrativa, con la loro idiomatica medietà stilistica, confermano il loro stretto legame con il genere epistolare, inteso in senso lato. Non si tratta solo di una sourapposizione, ormai definitiva, di sermones ed epistulae, che Orazio insegnava a distinguere, ma di un consapevole arricchimento del ternario, ormai aperto ai contenuti più svariati, e non soltanto alle alte forme liriche a cui lo avevano elevato prima Dante e poi Petrarca; forme che, come dimostra lo stesso Lattanzio, autore di una serie di Elegie in terza rima e di un capitolo di argomento sacro, continuano a essere praticate ma con la consapevolezza della loro eccezionale versatilità. Tale libertà espressiva, su un piano più ampio, rende un autore come Benucci difficilmente inscrivibile in una categoria storiografica netta e dimostra ancora una volta l'inapplicabilità, in assoluto, dell' etichetta di 'antipetrarchista'. ${ }^{\circ}$ Come per molti altri 'berneschi' di prima e seconda generazione, ${ }^{4 \mathrm{I}}$ scrivere sonetti 'petrarcheschi' e sonetti caudati, sonettesse, capitoli burleschi, rispetti, poesie sacre e cosi via, e addirittura includerli nella stessa raccolta era, banalmente, il modo più naturale di fare poesia.

Johnny L. Bertolio 
I. Cfr. G. Ballistreri, Benucci (Benuccio, Benutio), Lattanzio, in Dizionario biografico degli Italiani, 8, Roma, Istituto della Enciclopedia Italiana, I966, pp. 653-55 e M. A. Garullo, Notizie sulla vita e sull'opera di Lattanzio Benucci "giureconsulto sanese", in «Scaffale aperto», 5 (2014), pp. 9-48.

2. G. Ballistreri, Una corrispondenza poetica di Tullia d'Aragona, in «Il Mamiani», 3 (I 968), pp. 28-40.

3. S. Jacomuzzi, Nota in margine alla polemica Caro-Castelvetro: $i$ sonetti de "la civetta" di Lattanzio Benucci, in «Lettere italiane», 28.2 (1976), pp. 197-204.

4. Grosseto, Biblioteca Comunale Chelliana, cod. 7.

5. Su cui si veda K. Eisenbichler, The Sword and the Pen: Women, Politics, and Poetry in Sixteenth-Century Siena, Notre Dame, University of Notre Dame Press, 2012, pp. 224-32.

6. Beatrice Caetani era figlia di Bonifacio, duca di Sermoneta, e di Caterina Pio di Savoia e aveva sposato Angelo Cesi, signore di Monticelli, rimanendone vedova nel i 570 . Bonifacio Caetani, di simpatie francesi anche sulla questione di Siena, aveva partecipato, nel i 556, alla missione presso la corte di Enrico II voluta da Paolo IV e guidata dal cardinal Carlo Carafa, mentre Angelo Cesi combatté, sotto Pio V, contro gli Ugonotti in Francia. Beatrice, donna colta e versata nelle arti, specialmente nella musica, contribuì fra l'altro al completamento della Chiesa del Gesù a Roma (G. De Caro, Caetani, Bonifacio, in Diz̨onario biografico degli Italiani, r6, Roma, Istituto della Enciclopedia italiana, 1973, pp, 133-34, p. I33; A. Feves, Fabritio Caroso's Patronesses, in Women's Work: Making Dance in Europe before I800, ed. L. Matluck Brooks, Madison, The University of Wisconsin Press, 2007, pp. 49-70, pp. 55-58).

7. Siena, Biblioteca Comunale degli Intronati, P.V.44.

8. Felice Orsini, figlia di Girolamo, era andata in sposa, nel i 5 52, a Marcantonio Colonna, di simpatie imperiali: questi aveva militato contro Siena sia nel i 593 , per conto di Carlo V, sia nel i 594 al seguito delle truppe napoletane alleate di Cosimo de' Medici. Luogotenente di Giovanni d'Austria durante la battaglia di Lepanto, dal I 577 al i 584 fu viceré di Sicilia. Benucci poté entrare in contatto con Felice mentre questa si trovava a Roma, costretta a risiedervi con la suocera e le cognate sotto il pontificato di Paolo IV, ostile al Colonna (F. Petrucci, Colonna, Marcantonio, in Dizionario biografico degli Italiani, 27, Roma, Istituto della Enciclopedia italiana, I982, pp. 371-83, p. 372).

9. Siena, Biblioteca Comunale degli Intronati, H.VII.20.

ı. Roma, Biblioteca Angelica, I369, cc. 2r-5or e Biblioteca Apostolica Vaticana, Chig. I.VIII.295, cc. 433r-440r.

I I. Su questo tema fa da guida imprescindibile il capitolo (si perdoni il bisticcio) Una lettera in capitoli di S. Longhi, Lusus: il capitolo burlesco nel Cinquecento, Padova, Antenore, 1983, pp. 18 2-209. 
I 2. Un'eccezione è il Capitolo in vece de le sorti (F, cc. 2 Ior-2 I 2v+2 I $5 \mathrm{r}$ e Grosseto, Biblioteca Comunale Chelliana, cod. 8 , cc. $76 \mathrm{v}-80 \mathrm{v})$, pensato come alternativa spirituale all'uso profano di sorteggiare cartigli contenenti versi petrarcheschi la notte dell'Epifania; Lattanzio, come spiega nel relativo Avvertimento, compone invece massime ed esortazioni alla vita santa.

I 3. F presenta diversi tagli e lacune: non è chiaro a chi e soprattutto a cosa siano dovute queste mutilazioni a cui forse non furono estranee, visti i temi politicamente e religiosamente scottanti, anche ragioni di convenienza da parte di Lattanzio stesso in vista di una pubblicazione a stampa che comunque sarebbe stata già preceduta dalla divulgazione manoscritta dei testi.

I4. Il Capitolo de lo spedale, a la Signora Tullia d'Aragona e la Risposta a se stesso in nome della medesima Signora Tullia, in appendice (ma mutilo) a F. Bausi, "Con agra zampogna". Tullia d'Aragona a Firenze (I545-48), in "Schede umanistiche», n.s. 2 (I 993), pp. 6I-9I, pp. 85-9I e quello a Cosimo, sempre in persona di Tullia, in (col sottotitolo Su una prammatica sul vestir delle donne, fatta dal Duca) Poesie italiane inedite di dugento autori dall'origine della lingua infino al secolo decimosettimo, a cura di F. Trucchi, vol. 3, Prato, Ranieri Guasti, i 847, pp. 378-83.

I s. Sotto la direzione di Konrad Eisenbichler, è in fase di completamento da parte di chi scrive la tesi di PhD (Department of Italian Studies, University of Toronto), dedicata appunto alla vita e alle opere di Lattanzio Benucci.

\section{I6. Dedica cancellata.}

17. Il capitolo appare originariamente dedicato a Giulia Gonzaga ma è stato poi re-indirizzato a un'altra donna della famiglia, forse per la morte della donna o per ragioni di censura per via dei rapporti compromettenti di Giulia con i circoli valdesiani e in particolare con Juan de Valdés e Pietro Carnesecchi.

I8. La dedica originaria sembra essere per «Hippolita Gonzaga».

19. Cfr. Longhi, Lusus, cit., p. I 84.

20. Un antico Castello di Saltennano (o Saltemnano) tuttora si trova in Val d'Arbia.

2r. Fabriano e Colle di Val d'Elsa erano sede di numerose cartiere.

22. Poesie italiane inedite di dugento autori cit., pp. 383-84. Lo stesso rifiuto per questo formulario 'alto', associato al nome di Petrarca, si ritrova in Berni (cfr. Longhi, Lusus cit., pp. 2 I 8-19).

23. Cfr. Longhi, Lusus, cit., p. 197.

24. Il capitolo in questione si presenta senza dedica o, meglio, con una dedica $\mathrm{Al}$ Commun di Bibbiano ripudiata; presenta inoltre una serie fittissima di correzioni, dovute al cambio di destinatari, che tuttavia inducono a collocarlo, tanto nella prima quanto nella seconda versione, nell'orbita strettamente familiare essendovi citate, oltre alla madre, le sorelle dell'autore. 
25. Lezione originaria degli ultimi versi: «Del resto ogn'un di noi si trova sano; / io, com'ho detto, spero in tempo breve / rivedervi e baciarvi anco la mano. / Intanto io fo quel che 'l buon figlio deve».

26. 'Filastrocca' (e 'diceria') era diventato quasi termine tecnico per indicare un capitolo burlesco (Longhi, Lusus, cit., pp. 2 I 5-26).

27. Se il metro, il capitolo ternario, rimarrà imprescindibile anche dopo la prima fioritura di poesia burlesca, iniziata con Berni, con il tempo gli elementi linguistici di schietta fattura fiorentina, ritenuti veicolo di naturalezza espressiva, tenderanno a essere ridimensionati in seguito alla diffusione del genere tra autori non toscani (D. Romei, Berni e Berneschi del Cinquecento, Firenze, Edizioni Centro $2 \mathrm{P}$, I984, pp. 69-70 e ora in Da Leone $X$ a Clemente VII: scrittori toscani nella Roma dei papati medicei (I5I3-I534), Manziana, Vecchiarelli, 2007, pp. 225-26).

28. Si veda l'analisi del Capitolo de lo spedale condotta da Bausi, "Con agra zampogna" cit., pp. 79-82, sullo sfondo del discusso J. Toscan, Le carnaval du langage. Le lexique érotique de Burchiello à Marino (XV $V^{e}-X V I I^{e}$ siècles), 4 voll., Lille, Presse Universitaire de Lille, i98 I.

29. Per l'Aretino (debitore, in questo, verso l'ambiente rusticale senese) si veda P. Marini, "Piu pro fa il pane asciuto in casa sua...": formule proverbiali e sentenziose in Pietro Aretino, in Il proverbio nella letteratura italiana dal XV al XVII secolo. Atti delle giornate di studio (Università degli studi Roma Tre-Fondazione Marco Besso, Roma 5-6 dicembre 20I2), a cura di G. Crimi e F. Pignatti, Manziana, Vecchiarelli, 20I4, pp. 67-I I I (e il volume offre numerosi altri esempi), che osserva come l'utilizzo dei proverbi attraversi generi diversi, dominando in particolare le opere comicosatiriche e le lettere.

30. Si dice «di chi sta per qualche accidente male, o d'animo, o di corpo, per essere il pollo pesto propria vivanda de' gravemente ammalati» (Vocabolario degli Accademici della Crusca, Venezia, Giovanni Alberti, r6 г 2, p. 634).

3I. Questi volatili erano così legati ai campanili (come i fiori alla primavera e le stelle al cielo notturno) che l'espressione 'cornacchione di campanile' era usata per indicare «chi non si lascia aggirare, e muovere da parole» (Vocabolario degli Accademici della Crusca, Venezia, Giovanni Alberti, i6 1 2, p. 226: cfr. il capitolo $A$ le sorelle, c. I $23 \mathrm{v})$.

32. Espressione scherzosa per indicare l'atto del bere (cfr. Vocabolario degli Accademici della Crusca cit., p. I I 6), qui con valore metaforico, come in quella, parallela e attestata dal medesimo Vocabolario, di 'mettere il becco in molle', ovvero 'iniziare a chiacchierare senza costrutto' (come nel capitolo mutilo a Virginia Martini Salvi, c. I 2 Ir).

33. Longhi, Lusus, cit., p. 224.

34. Cfr. S. Bongi, Il velo giallo di Tullia d'Aragona, in «Rivista critica della letteratura italiana», 3.3 (1 886), pp. 8 5-95, pp. 88-90; G. Masson, Cortigiane italiane del Rinascimento, trad. it. S. Sudrie, Roma, Newton Compton, I98 I, p. I62; Bausi, "Con agra zampogna", cit., p. 69. 
35. S. Bongi, Documenti senesi su Tullia d'Aragona, in «Rivista critica della letteratura italiana», 4.6 (I 887), pp. I86-88; Masson, Cortigiane italiane del Rinascimento cit., p. I 52 .

36. Bausi, "Con agra zampogna", cit., pp. 69-70.

37. Bausi, "Con agra zampogna", cit., pp. 79-82.

38. Come avveniva negli scambi di sonetti (Longhi, Lusus, cit., p. I97).

39. Essendo l'autografo mutilo (come pure la trascrizione fattane da Bausi), integriamo le ultime terzine con il ms. Chig. I.VIII.296 (c. $258 \mathrm{r}-\mathrm{v}$ ).

40. Tra i vari lavori sul tema si vedano in particolare Longhi, Lusus, cit., p. 2 I 9 e i contributi di R. Gigliucci, Antipetrarchismo interno o petrarchismo plurale? e P. Procaccioli, Pietro Aretino sirena di antipetrarchismo. Flussi e riflussi di una poetica della militanza, in Autorità, modelli e antimodelli nella cultura artistica e letteraria tra Riforma e Controriforma. Atti del Seminario internazionale di studi (Urbino-Sassocorvaro, 9-II novembre 2006), a cura di A. Corsaro, H. Hendrix e P. Procaccioli, Manziana, Vecchiarelli, 2007, pp. 91-101 e 103-29.

4I. La prima edizione del Primo libro dell'Opere burlesche di M. Francesco Berni, di M. Gio. della Casa, del Varchi, del Mauro, di M. Bino, del Molza, del Dolce, et del Firenzuola, a cura di Anton Francesco Grazzini, risale al i 548 (Firenze: [Bernardo Giunti]), ma fu ristampata nel i 550 , nel 's2 e nel 's 5; e la princeps del Secondo libro, con testi di M. Francesco Berni, del Molza, di M. Bino, di M. Lodovico Martelli, di Mattio Francesi, dell'Aretino e di diversi autori fu pubblicata, sempre presso i Giunti, nel i 555 (Longhi, Lusus, cit., pp. 25-28). 


\section{APPENDICE}

Per la loro esemplarità ai fini del discorso condotto supra, sono stati trascritti i due capitoli ( $F$, cc. I 2 r-25r, numerati «4» e « 5 » dall'autore) dedicati da Lattanzio Benucci alla madre, la senese Girolama Campani, a cui le fonti erudite attribuiscono non irrilevanti qualità poetiche e che, a Roma, fu in contatto con importanti famiglie aristocratiche. Essendo utile ai fini dell'interpretazione del testo, è stato inoltre aggiunto un essenziale apparato variantistico (s'intenda: delle varianti intelligibili) in coda a ciascuno dei due capitoli.

I.

A la nobilissima Madonna Girolama Campani de’ Benucci. Capitolo

Non posso più tener la penna a freno, ché con essa convien ch'io vi favelli, perch'ho di troppe cose il capo pieno.

Ma non pensate ch'i soggetti belli sien cantati da me né che le carte v'empia di "unquanchi", di "soventi" o "snelli":

son rozzo, come ben sapete, e parte non hebbi mai tra le nove sorelle, ma seguo la natura e fuggo l'arte.

Hor benché scarse a me tutte le stelle in ciò sien state, in ogni modo io voglio dirvi quattro parole, o brutte o belle.

Ho gran fastidio e senza fin mi doglio non haver tempo a mio modo, ché certo non bastarebbe questo e un altro foglio.

Vedendo partir voi, madre, ho sofferto gran travaglio e col senso la ragione più volte ha combattuto a campo aperto: quel primo ogn'hor mi preme e dà di sprone che per voi lassi anch'io queste contrade; l'altra vien poi, ch'a restar mi dispone. ${ }^{\text {I }}$

Né mi risolvo ancor, ma perché rade volte dell'huom con prestezza il parere o la sentenza in dritta parte cade, 
io m'ho pensato squadrare e vedere più d'un giorno e più d'una settimana se venir debbo o 'l piè qui ritenere.

Credo che mi parria pur cosa strana partirmi e non so ben com'io potesse lassar qui di Mambrin la cieca alfana; ${ }^{2}$ né contento sarei s'io non vedesse ch'a lui n'andasse, perch'una tal razza sarebbe mal se con altrui si stesse.

Impazzo anch'io con questa bestia pazza, c'ha sempre intorno un branco di cicale, o stia su l'uscio o vada al forno o in piazza,

che dicon senza fine, e dicon male; ma pensatevi pur ch'ella ciascuna dell'altre avanzi e sia la principale.

Io non potrei di mille parti l'una de' fatti suoi contarvi, ancor ch'a voi poche ne sian celate o ver nessuna.

Onde dovrei tacere; ma da poi ch'a le mie spese qualcuna n'imparo, dironne, acciò pietà n'habbiate a noi.

Qui verrebbe a bisogno un stil più raro per dir con quant'industria l'insalata ella ci assetti, al ciel fosco et al chiaro.

O herba sopra tutte avventurata, ${ }^{3}$ degna che di te sempre si ragioni, che manchi col terren dove sei nata!

Io lassarei per te starne e capponi, quand'a guisa di solcio io ti discerno, piena di porcelletti e lumaconi.

Questo cibo a me par che sia moderno, né tal cred'io che s'apparecchi dove mangia il fiero signor del lago Averno; tal che bisogna, a voler che ne giove, chiudere gli occhi e come medicina cacciarla giù perché non vada altrove.

Hor lasso questa e passo a la cucina, ch'a modo usato par l'ordine istesso, o vogliate da sera o da mattina,

e, poi ch'in quest'intrico io mi son messo, 
riponendo da parte ogn'altra cosa, vengo a dir sol come si faccia il lesso:

minestra delicata e saporosa, anzi più tosto vera incennerata, u' la povera bestia sta nascosa.

Credo le paia d'essere in bucata, né si deve per questo lamentare, essendo in altra parte ristorata, perch'io l'ho vista in la pignatta entrare asciutta e intera, onde 'l cane e la gatta da tal cosa si vogliano appellare.

Hor chi potria mai dir come s'adatta a far per casa tutte le faccende, che par da tre dì in qua tutta rifatta?

Io ne saprei contar cose stupende, ma perch'altrove, come voi sapete, n'ho scritto, questo basti a chi l'intende; ${ }^{4}$ voi queste insieme con quelle aggiugnete e pensatevi poi che tutte in opra hora le metta e così la correte.

Ma costei lasso e voglio dir hor sopra i fatti nostri almen trenta parole, prima che 'l sonno gli occhi mi ricopra.

Vorrei saper s'ancor la sorte vuole c'habbiate costì gente forestiera e se per ciò a riguardo star vi duole.

A me par certo una strana matera a veder a rovescio andar il mondo: vorrei tornasse sì come prim'era.

Quand'io ci penso, tutto mi confondo e per questo vi dico apertamente il mio parer, ch'a gli altri poi nascondo.

Di più, perch'io son molto diligente, vorrei saper da voi come si stia il mio vicino, il compagno e 'l parente.

Io temo ogn'hor di qualche nuova ria, però che mi vien detto il mio cugino esser costì, che mi par gran pazzia.

M'ha detto ancor non so qual contadino ch'in questa settimana in che noi siamo 
sarà ricolto tutto quanto il vino;

ond'io che pur mangiar dell'uva bramo,

vi prego n'ammaiate il mappamondo,'

acciò co i tordi poi ce la godiamo.

Se voi dicessi c'hora io mi confondo, c'ho detto di non esser resoluto, hor dico di venire, io vi rispondo

ch'io sto in cervello e non mi son perduto, ché s'io non vengo per fermarmi, penso venir per quattro giorni, ch'è dovuto.

Intanto voi per dar qualche compenso a quest'assenzia mia, le mie sorelle salutate per me con ogni senso.

Ditele ch'io vorrei saper novelle di loro e che mi scrivin qualche volta, né mi curo che sian parole belle.

Hor io vo' fare un'altra giravolta e, lassando da parte i casi nostri, tesser una gran tela c'ho ravvolta.

Perciò date voi tregua ai pensier vostri e prestatemi tutta l'attenzione, se volete ch'a pieno io ve la mostri.

Io viddi il nostro amico hieri in giubbone schietto, com'una spada rugginosa, che gli par esser da più ch'un garzone,

e tanto più perch'hora ha la sua sposa gentil mandata a stazzonare altrui, ${ }^{6}$ onde solo e contento hor si riposa,

sì che vedete che huomo è costui; io per me ne stupisco in pensar solo com'esser può che mai si pensi a lui.

Mi fece molte carezze e "Figliuolo», mi disse, «va', che tu sii benedetto», ma nel donarmi già non corse a volo;

e certo il fatto suo parmi un dispetto, ch'a tutti ogn'hor buone parole dia, poi tenga per la pari in man sì stretto.

Ma sarebbe la mia gran frenesia, s'io m'occupassi fin a domattina in quest'impresa co' la fantasia. ${ }^{7}$ 
Hor perché 'l fin del mio dir s'avvicina, vi vo' dar nuove di noi stessi ancora, che facciamo una vita paladina;

e benché così sia, non è ch'ogn'hora non ci paia mill'anni che torniate e appena sete de la casa fuora.

Ma voi di ciò non vi maravigliate; ché, mentre sete in paese lontano, non posson qui star liete le brigate.

Io sanato non so', ma son ben sano, e spero ancor di rivedervi presto e come profumato cortigiano

la man baciarvi: allhor saprete il resto.

\section{Apparato critico delle lezioni originarie}

I 8 campo] viso 95 a voi] vi 127 nostro amico] padre vostro I 32 hor si riposa] homai si posa I $34-35$ in pensar solo / com'esser può che mai si pensi a lui] e penso solo / com'esser può siate nata di lui i 53 non posson qui star liete le brigate] come posson star liete le brigate? i 54-57] Del resto ( $>$ basta ch') ogn'un di noi si trova sano; / io, com'ho detto, spero in tempo breve / rivedervi e baciarvi anco la mano. / Intanto io fo quel che 'l buon figlio deve. > Io sanato non so', ma son ben sano, / e spero anchor di rivedervi in breve / e come profumato cortigiano / la man baciarvi e far quanto si $\mathrm{d}<$ eve $>$.

\section{II.}

\section{A la medesima. Capitolo}

Poi ch'io son fuor dei libri una mezz'hora

e so ch'io mi starei a ingiardinare ${ }^{8}$

intorno ai fatti de la mia signora

s'io non pigliassi altra faccenda a fare, mi son per questo in tutto resoluto

d'haver con voi un pezzo a ragionare.

So ben che voi direte che venuto mi sia nel capo il grillo; hor vi rispondo che caro m'è che ciò vi sia creduto,

perché farà la scusa a tutto il mondo, che mi sia stato forza di sfogarlo, se ben con il mio dir non pesco al fondo.

Ma vogliam grillo a capriccio chiamarlo:

egli ha tanto poter nel capo mio, che, com'ho detto, è forza seguitarlo. 
A darci dentro e 'l primo sarò io; voi sgombrate del petto ogni pensiero, che vi rendesse l'animo restio.

Io per farvi sapere il chiaro vero dei casi miei, son dato in preda al duolo e mi struggo, m'ammazzo e mi dispero;

ché se lo stare in un buon letto9 solo è gran tormento, hor come solo in Siena potrà contento star vostro figliuolo?

E mi viene a pensarci sì gran pena, ch'io sto per disperarmi a fatto a fatto o per trovar de la pazzia la vena; ch'almanco, s'io pur divenissi matto, a tutto il mondo restarei scusato, ond'uscirei d'intrigo al primo tratto, perch'andarei scorrendo in ogni lato e mi trarrei di belle fantasie, ch'ogn'un direbbe: «Lassa, egli è impazzato».

$\mathrm{E}$ in ogni modo tante astrologie non ti servano ad altro ch'a levarti dei contenti e piacer tutte le vie.

Attendi a le sentenze, attendi all'arti, ti bisogna esser asin di te stesso, se tu da qualche cosa vuoi pur farti.

S’io non mi fussi a questa impresa messo, non mi ci metterei, però che vane son le fatiche et ben lo veggo espresso;

e per non dover poi pensare al pane e per fuggir lo stento amore Dei, passando me l'andrei d'hoggi in domane.

Almen quella è una vita de le sei e tal ch'io non le trovo concorrenza, sì che ben volontier me la torrei, se già non fusse contra conscienza il godersi pel verso questo mondo, lodando sempre a gli altri l'astinenza.

Hor dica pur chi vuole, io non rispondo e ficco il capo innanzi a senno mio, ché facendo altrimenti sarei tondo.

Ma lassiam questi, perché al fine il fio 
paga ciascun dell'ozio, et però voglio tornar col capo al primo lavorio; ${ }^{10}$

e farò forza a me perch'io non soglio parlar di queste genti per sì poco, ma hor mi vo' star queto come l'oglio.

Io so' spolpato et ogn'hor più mi cuoco, perché Amor dentr'al mio petto ha nascosto un vivo, ardente et invisibil fuoco;

e quanto più dall'esca io son discosto, tanto maggior la fiamma provo allhora, che quand'appresso me le trovo posto.

La mia disgrazia vuol ch'io ne sia fuora ancor per qualche giorno, ché saranno i giorni un anno e un mese ciascun'hora.

S'al suo ritorno pur cessasse, il danno portarei in pace, ma conosco chiaro ch'oltra la mala Pasqua, harò il mal anno.

Cupido del ben mio pur troppo avaro, tu vedi il mio dolore e non lo curi, anzi t'è 'l mio languire e 'l dolor caro.

Ghiotto da forche, tu dal mio cor furi ogni dolcezza, ogni contento e gioia, empiendol di pensieri atri et oscuri,

e per accrescer poi questa mia noia, son fuor d'ogn'altro spasso, acciò ch'in breve possa in me tanto il duol ch'io me ne muoia.

Già mentre ero costì, la pena greve mi si facea men dura, et era come al fuoco cera et al sol fredda neve.

Hor son gravato di sì fatte some, ch'io non paio più desso e m'è restato di quel di prima solamente il nome.

Ma non pensate che mi sia cascato, con tutto questo, de la mente mai il bel tempo che già costì m'ho dato; e tanto più ch'essendo hor pien di guai è forza ch'io ci pensi tuttavolta, a me stesso dicendo: «Hor che farai?».

Né paia questo strano a chi m'ascolta, ché se trova il notar che se ne roghi, 
gli dirò la sentenza c'ho raccolta:

la lunga assenzia, il veder varii luoghi, praticar altre femmine di fuore par che sovente disacerbi e sfoghi

dell'amorose passioni il core.

Ell'è pur vera e ne fo prova adesso, mercé di chi mi strazia a tutte l'hore.

Io mi vo ricordando bene spesso de la stanza piacevol di Menzano, mentre lo starvi già mi fu concesso.

Amor allhor mi lusingava in vano, col dirmi «Piangi, doloroso e lasso, poi che da la tua diva sei lontano; muovi presto di qui, deh, muovi il passo, cerca di riveder quel suo bel volto!»; ch'io rispondevo allhor: "Vattene a spasso!

Hora sì ben ch'io sarei sciocco e stolto s'io tentassi di far più duro il giogo, potendo qualche dì viverne sciolto».

Maraviglia non è dunque se il luogo dei miei diletti mi sta ne la mente e se col ricordarmene mi sfogo.

Perch'ancor voi, se vi darete mente, vedrete ch'ell'è cosa naturale, non solo in me, ma posta in ogni gente.

La nostra alfana, ${ }^{\text {I }}$ che pur poco vale, quando torna costà, va con tal fretta, che sarebbe disdizio havendo l'ale.

Infin cotesto luogo ogn'uno alletta e come i tordi impania le persone; dunque è ben dritto s'hor sì mi diletta, oltra che io ci ho su molta ragione, poi che costì vi feci patir pena: voi m'intendete pur per discrezione. ${ }^{\mathrm{I}}$

E se ben poi me ne sto sempre a Siena, pur com'io sento Menzan ricordare, mi trovo l'alma di dolcezza piena.

Né so quel ch'io m'andassi anno a pescare col dirne male fuor d'ogni dovere, ma me ne voglio un giorno anco appellare; 
ch'io non havevo allhor dritto vedere, come ne posso produr testimoni, non uno o due, ma filze intere intere.

Hor addunque mi reco ginocchioni

e chieggo perdonanza del mio fallo, sperando pur ch'al fin mi si perdoni: non vo' contra il dover mai fare il callo.

Hor lassiam questo che n'ho detto assai e seguitiamo il cominciato ballo.

Da poi ch'ultimamente io vi lassai, visto il bel tempo che s'era avviato, ho sentita maggior pena che mai.

Se venni a Siena tutto spennacchiato, chi m'havesse veduto pel traverso, harebbe detto: «Egli è trasforamato!». ${ }^{{ }^{3}}$

Non so' smarrito, no, ma ben disperso per le ragion che già sopra v'ho dette, ne la malinconia fitto e sommerso.

Hor s'io ho spesso di sì fatte strette senz'haver pur un spasso d'un quattrino, credo ch'io tirarò su le calzette

e non varrà poi dire: «Il poverino s'è morto e non faceva male al pane ch'ei calpestava et era me' del vino».

E benché queste sien pur cose strane, bisogna star contento di sua sorte, ché le nostre fatiche in ciò son vane.

Ma poi c'ho ad esser preda de la morte, vo' dir al mondo il mio parer fuor fuore e s'udir non mi vuol gridarò forte:

«Egli ha poco cervello un che si muore».

Pur poi che bisogna irne a suo dispetto, vorrei poter innanzi all'ultim'hore

haver colei che m'arde a mio diletto, acciò che meco ell'havesse a bruciare, né fusse sciolta s'hor mi tien sì stretto,

et a mio modo vestire e calzare, dormir quant'io volessi a mio piacere, mandar i tristi a bastonare il mare, quand'io n'ho voglia ancor mangiare e bere, 
gire a cavallo come un paladino,

star a mia posta hor dritto, hor a sedere,

haver anco a la man qualche carlino,

esser amato da le donne belle

e farmi a tutte quante schiavolino,

lassar andare il contemplar le stelle

col cercare i segreti di natura,

e studiar solo in sul Cento novelle,

tener in ogni impresa la ventura,

starmi costì con voi a trionfare

da la vendemmia insino a segatura.

Oh io mi crederei che tempo dare!

Ma non m'avveggo ch'io sono imbarcato

e non l'harei creduto di sognare.

Hor fate conto io non n'habbia parlato, per non parer un sacco senza fondo, e riduciamla a un dir più costumato.

Vorrei dunque godermi questo mondo, non tante cose, e trovarmi con voi, a far la chiarenzana e 'l ballo tondo.

Quest'è quel poco ch'io vorrei, ma poi che mi si vieta, io maladico il fato e la fata Morgana e i fabbri suoi,

e vo temprando il mio infelice stato con la memoria del tempo felice, che mai non viddi e mai non mi fu dato.

Ma poi ch'ogni piacer mi si disdice, vi ricordo impariate a le mie spese, come 'l volgar proverbio ogn'hor vi dice.

Voi starete costì quest'altro mese:

pigliatevi il bel tempo, ché potete, e fate sopra tutto buone spese.

Me con voi sempre nascosto haverete, ma se cercate bene io vi prometto che veramente mi ci troverete.

Non dirò già che con voi venga al letto, però ch'allhor sarà la compagnia forse sì stretta ch'io non mi ci metto.

Ma quando voi sarete per la via con coteste sgherracce di Menzano 
a far il ballo, io pigliarò la mia

e strignerolle a mio modo la mano, sia chi si vuol, ch'io non vi porrò cura, per ch'ogni cosa io vo' mettere a piano.

Quand'uscirete poi fuor de le mura per andar verbigrazia al Castiglione, ${ }^{\mathrm{I}}$ vi sarò dietro e vi farò paura;

né mi potranno veder le persone, c'ho tolta l'elitropia a Calandrino $^{\text {Is }}$ e potrò farlo senza discrezione.

Se la fante ${ }^{\mathrm{I}}$ andarà sola pel vino, o veramente qualc'altra persona, io le nasconderò tutto il tuppino.

Quand'andarete al Boschetto, a Cantona ${ }^{17}$ innanzi giorno, io mi porrò in agguato e vi torrò s'harete cosa buona.

Voi non potrete andare in alcun lato, perché per tutto vi vo' seguitare, da uno instinto natural tirato;

sì che fatevi pur tutte ciurmare, e fate per le case l'avventario, ch'io le vo' tutte scopare e furare,

e serrate ogni cassa et ogni armario, ch'io torrò paneruzze da cucire e ritrovarò tutto il calendario.

Non già da ver, ma sol per farvi dire, torrò dell'uscio a qualcuna la chiave per vederla poi meglio schristianire.

Né vo' che ciò vi si dimostri grave, ché sapete ch'io scherzo volontieri e m'è questo burlar grato e soave.

Hor mentre ch'io vi scuopro i miei pensieri, vorrei, con sicurtà vel dico in rima, che mi facessi un mondo di piaceri, tra $i$ quali il primo che si siede in cima è ch'io sia in grazia da mattino a sera di chi tanto a ragion se stessa stima.

Questa non mi par certo cosa vera, c'habbia da esser madre così ratto, e pur ha già di figliuoli una schiera: 
et io che son un gioven così fatto, et ho di lei più tempo e più persona, son senza rede e non ne fo contratto.

A dir dell'altre hora il desir mi sprona, ma non m'arrischio et ho tanta paura, che sì e no nel capo mi tenzona; ${ }^{18}$

e però me ne vo lungo le mura e così cerco il resto del Castello, e follo a tutte quante a la sicura saper com'io l'ho fitte nel cervello, ch'io le vo' mille moggia di quel bene, che dee volere un marito e un fratello.

Ma qui bisogna rimirar ben bene ch'io non parlo di quelle che potrieno esser mie madri, e non saria già bene,

ch'io per me tutte queste vo' che sieno reverite da madri e niun le tocchi, perch'elle han di consigli il capo pieno.

Ma già mi sento calar dentro a gli occhi una certa renetta, ond'affatica posso tener che 'l sonno non m'imbrocchi;

e però sarà ben che tosto io dica quel ch'io vo' dire e licenziar di poi la Musa ch'io mi tengo per amica.

Onde di nuovo a tutte quante voi, donne, mi raccomando di buon core, che 'l Cielo mi mantenga ogn'hor con voi.

Io sono schiavo a tutte e servitore, salve le fedi, ch'io son qua intigito, ${ }^{19}$ come la dama mia torna di fuore.

Annibal nostro, ${ }^{20}$ de la mente uscito non m'è, se bene io non lo veggo ogn'hora e gli porto un amor più che finito.

Ma ci sarebbe da dire ancora un'hora; però fo qui fardel di tutto il resto e m'offerisco a tutti questi ancora.

Io spero un dì di rivedervi, e presto, ch'io vo' far qui qualche stramanceria, che non si truovi scritta nel Digesto, acciò che 'l nostro Capitano harpia ${ }^{21}$ 
mi confini costì per sempre mai, ond'in eterno obligato io gli sia.

Hor io vi lasso e ritorno a' miei guai:

pregate Dio per me ch'io non mi muoia,

ché ne sarebbe danno pur assai;

me ne vo in letto a distender le cuoia.

Apparato critico delle lezioni originarie

9 ciò vi] questo I 2 ben con il] con questo i6] hor diamci dentro <illeggibile> I 8] che vi premesse et ogni stran' desio 3 I perch'andarei] andarei poi 62 ] però che dentr'al petto è ancor nascosto 63 un] quel 80 altro spasso] conforto io8 diva] donna I Io suo bel] chiaro I 8 darete] porgete I 27 oltra] da poi molta]tanta I 28 poi che] ché già I 49 veduto] mirato I 5 I ben disperso] in tutto perso I 7 I tien si] tiene $20 \mathrm{I}$ che mai non hebbi e mai mi fu mostrato 
I. Piuttosto che all'allontanamento della madre e delle sorelle, sembrerebbe più verosimile pensare a un soggiorno di Lattanzio in campagna, presso i nonni materni, magari per motivi di salute, forse a Menzano, nominata nel capitolo successivo. Del resto, anche la madre appare ora ritirata in «paese lontano», e non è certo se si tratti di Siena.

2. Espressione proverbiale e scherzosa (qui unita a «cieca») che indica una donna alta, di cui tuttavia ci sfugge l'identità precisa, a meno che non si tratti della nonna materna.

3. Di simile argomento, in negativo, parlerà Lattanzio nel capitolo contra il cavolo.

4. Non sappiamo se qui Lattanzio alluda a qualche capitolo perduto o a qualche lettera personale inviata alla famiglia.

5. 'Decoriate con festoni [d'uva] tutto il mondo': sembra questo il significato più plausibile dell'espressione idiomatica.

6. Se Lattanzio sta qui parlando del nonno materno, lasciato solo dalla moglie, allora è possibile che sia la madre di Girolama la «cieca Alfana» che sta tormentando Lattanzio e il suo stomaco.

7. I vv. I 43-44 sono riscritti su una illeggibile versione originaria, che poi prosegue, sempre cassata, per altre tre terzine non sostituite.

8. 'Mi metterei a [pensare alla mia signora]' (cfr. A. Castellani, Il "Vocabolario sanese" del fondo Biscioniano della Biblioteca Naz̧ionale di Firenze, in "Lingua nostra», 7.3-4 [i 947], pp. 65-78, p. 72).

9. Sotto questa lezione definitiva e chiaramente autocensoria si intravede il nome del luogo in cui Lattanzio si trovava e che purtroppo è illeggibile (sembra iniziare per R- e in quel caso la candidata sarebbe «Roma», seguita da un'altra parola di due sillabe o da due monosillabi).

ı०. Queste terzine sono particolarmente tormentate: la lezione originaria risulta di difficile lettura.

I I. Allusione alla donna già evocata nel capitolo precedente.

I 2. Accanto a questa terzina una nota autografa rileva: «Natività dell'autore»: Lattanzio sarebbe dunque nato a Menzano, presso Casole d'Elsa.

I 3. 'Ferito' o, più crudamente, 'forato' (forte idiotismo senese).

I4. Anche qui si intravede una lezione originaria con un probabile agiotoponimo $(«$ San $\mathrm{M}-»)$ di difficile interpretazione.

I 5. Cfr. Dec. VIII 3.

i6. La lezione originaria, completamente cancellata, potrebbe nascondere un nome proprio di persona. 
I7. Fuori Casole d'Elsa esistono tutt'oggi due località ('poderi') così chiamati.

I 8. Purg. 8, i I I.

I 9. 'Sequestrato', 'pignorato' (variante toscana, e specificamente senese, di ‘staggito': cfr. Castellani, Il "Vocabolario sanese" cit., p. 72).

20. Se Lattanzio, come pare probabile, ha scritto questo capitolo da Roma, si potrebbe trattare di Annibal Caro, di cui era amico.

2I. Probabile allusione a qualche funzionario (romano o senese) ostile a Lattanzio e quindi in grado di confinarlo fuori città, in particolare presso Casole d'Elsa («costi») ove la madre si trova ora. 